\title{
Wollemia nobilis (Araucariaceae) - Von einem der seltensten australischen Endemiten zur Massenpflanze
}

Hubertus Nimsch \& Veit Martin Dörken

\begin{abstract}
Wollemia nobilis (Wollemi Pine) was discovered in the Blue Mountains of Australia in 1994. Wollemia nobilis is regarded as a living fossile. Since a few years trees are sold by specialist nurseries. The history of discovery, morphology and ecology are outlined. In addition available experience regarding the winter hardiness with specimens grown in Germany are sumarized.
\end{abstract}

\section{Zusammenfassung}

Wollemia nobilis (Wollemi-Kiefer) wurde erst 1994 in Australien in den Blue Mountains entdeckt. Die Art gilt als lebendes Fossil. Seit einigen Jahren sind Wollemia-Bäume auch bei uns im Fachhandel erhältlich. Entdeckungsgeschichte, Morphologie und Ökologe werden vorgestellt. Außerdem werden Erfahrungen zur Winterhärte mit in Deutschland ausgepflanzten Exemplaren zusammengefasst.

\section{Einleitung}

Kaum eine andere Baumart hat in den letzten Jahrzehnten für so viel Aufsehen gesorgt wie die australische Wollemia nobilis. Auch wenn die deutsche Bezeichnung Wollemi-Kiefer oder im
Englischen Wollemi Pine dies nahelegt, ist diese Baumart nicht näher mit den echten Kiefern (Pinus, Pinaceae) verwandt. Wollemia gehört mit zwei weiteren rezenten Gattungen (Araucaria und Agathis) zur erdgeschichtlich sehr alten Familie

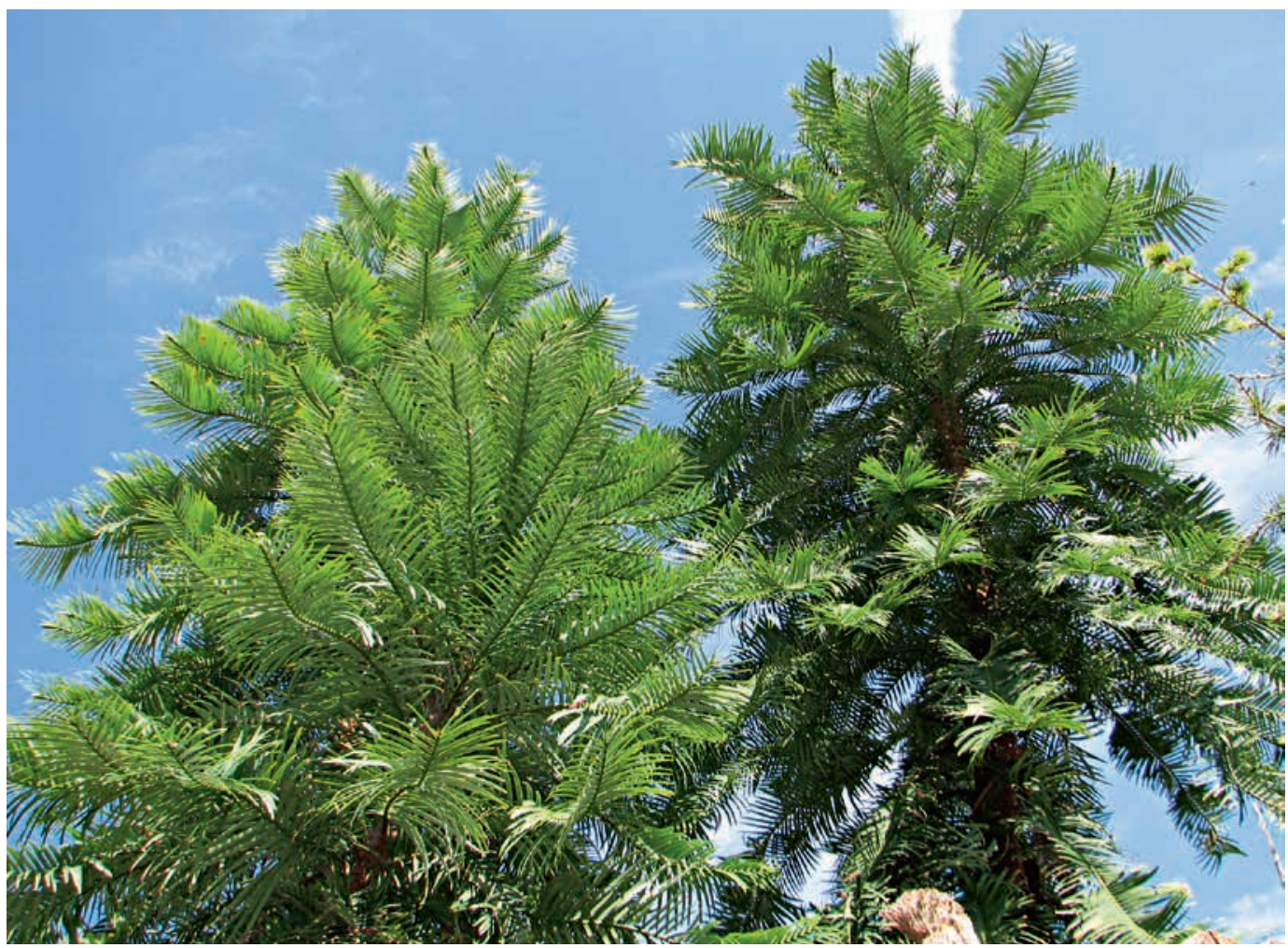

Abb. 1: Kräftige Exemplare von Wollemia nobilis. (Foto: V. M. DöRkeN) 
der Araucariaceae (Araukariengewächse). Die Gattung Wollemia galt als seit rund 65 Millionen Jahren ausgestorben (Nimsch 2011 a). Daher erwies sich der Zufallsfund im Jahre 1994 von einigen lebenden Wollemia-Individuen in einer unzugänglichen Schlucht in den Greater Blue Mountains, etwa $200 \mathrm{~km}$ westlich von Sydney, als eine wahre botanische Sensation. Die rezente Art Wollemia nobilis unterscheidet sich kaum von den bis dato bekannten kreidezeitlichen Fossilien vom australischen Kontinent (TAYLOR et al. 2012). Paläobotaniker vermuten sogar, dass die Art bzw. deren direkte Vorfahren bereits vor 200 Millionen Jahren zur Zeit der Dinosaurier existierten. Daher wird Wollemia häufig auch als der Dinosaurier unter den Pflanzen bezeichnet.

Auf darauffolgenden botanischen Exkursionen in das Fundgebiet wurden noch etwa hundert weitere Individuen gefunden. Bemerkenswert ist die Tastsache, dass alle gefundenen Individuen identisches Erbgut aufweisen und somit Klone darstellen. Selbst die durch sexuelle Vermehrung entstandenen Sämlinge zeigen keine genetische Variation in der Erbinformation im Vergleich mit deren Elternbäumen (Woodford 2005).

Diese Tatsache macht das Überleben der Wollemia nobilis über einen solch langen erdgeschichtlichen Zeitraum noch ungewöhnlicher, geht man doch allgemein davon aus, dass genetische Vielfalt generell einen entscheidenden Selektionsvorteil darstellt. Und die Bedrohung der Population wird nicht geringer. Das aktuell wütende australische Megafeuer brennt auch im Wollemi-Nationalpark. Ein schwerer Brand am Wuchsort der Wollemia nobilis könnte im schlimmsten Fall zur Ausrottung der kleinen Relikt-Population dieser urtümlichen Konifere führen.

Warum alle aufgefundenen Individuen genetisch identisch sind, ist noch nicht geklärt (Woodford 2005). Zum Erhalt dieser sehr seltenen Art wurde mit einem massiven Erhaltungskulturprogramm begonnen. Die Art wurde sowohl traditionell über Samen und Stecklinge als auch im Labor durch Gewebekulturen vermehrt, sodass

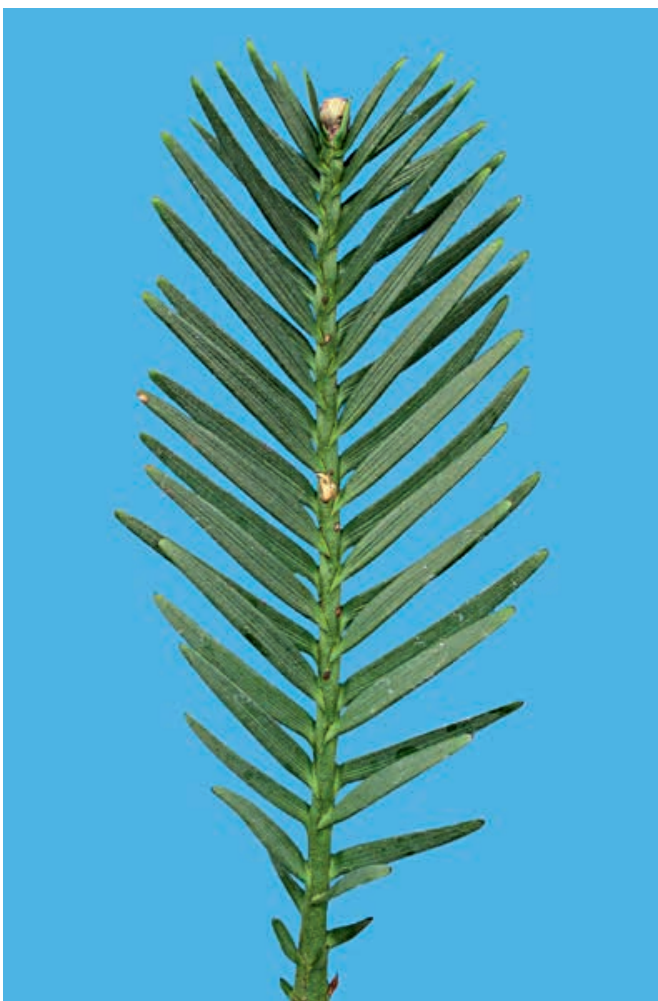

Abb. 2: Zweig von Wollemia nobilis. (Foto: V. M. Dörken)

sie heutzutage zu erschwinglichen Preisen sogar in Gartencentern angeboten wird.

\section{Systematik}

Araukariengewächse, zu denen auch Wollemia gestellt wird, sind bereits aus der späten Trias vor ca. 215 Mio. Jahren bekannt (FARJon 2008). Die Gattung Wollemia umfasst nur die einzige Art W. nobilis W.G. Jones, K.D. Hill \& J.M. Allen. Wollemia war vor rund 100 Millionen Jahren an der Bildung enormer Waldbestände maßgeblich beteiligt und ist fossil recht gut dokumentiert. Bis zu ihrer Wiederentdeckung im Jahre 1994 ordnete man den Araucariaceae nur die zwei Gattungen Araucaria und Agathis zu. Vertreter letzterer Gattung sind bei uns nicht ausreichend winterhart und deshalb weniger bekannt. Nur die chilenische Araucaria araucana ist unter unseren hiesigen klimatischen Bedingungen vielerorts winterhart und in vielen Gärten ein beliebter Solitärbaum. 


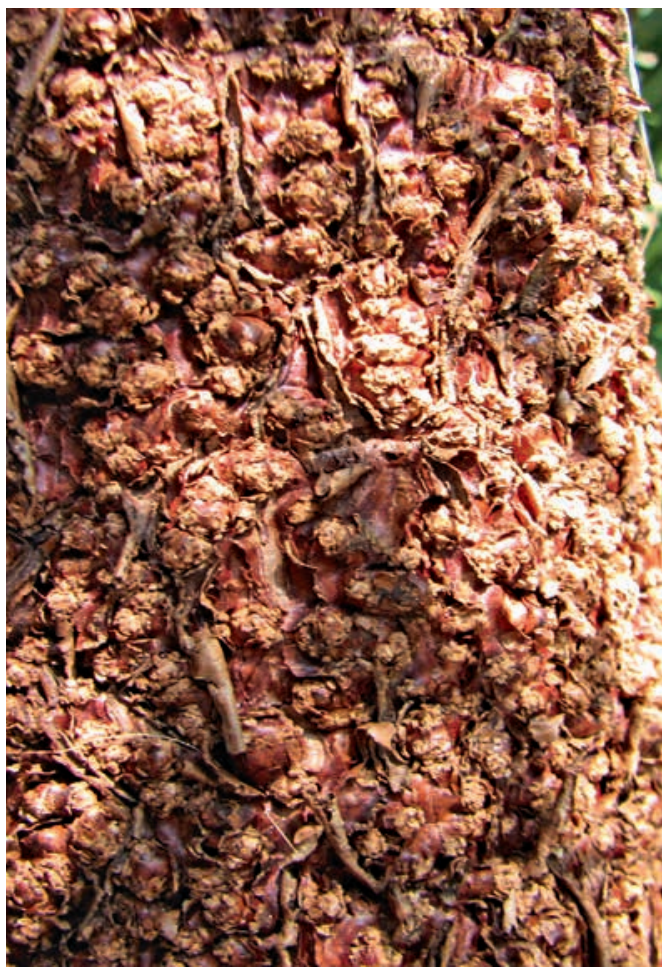

Abb. 3: Die Borke älterer Bäume ist von vielen kleinen Blasen überzogen. (Foto: V. M. DörkeN)

\section{Die Wiederentdeckung}

Heutzutage ist es allgemein akzeptiert, dass Wollemia die dritte Gattung der Araukariengewächse ist. Im Jahre 1994, als der Ranger David Noble im heutigen australischen Wollemi National Park in einer tiefen, unzugänglichen Schlucht mehrere große, ihm unbekannte Bäume fand, war dies alles andere als klar. Es war der Beginn einer Geschichte, die als eine der größten botanischen Sensationen des 20. Jahrhunderts bezeichnet werden kann. Erst nach einiger Zeit und dem Besuch einiger Botaniker vor Ort war klar, dass es sich um eine urtümliche Pflanze handelte, die seit über 65 Millionen Jahren als ausgestorben galt. Die Bedeutung dieser Entdeckung kann mit einem Fund eines noch lebenden Dinosauriers verglichen werden. Durch fossile Pflanzenteile von Wollemia ist belegt, dass es vor ca. 100 Millionen Jahren riesige Waldbestände dieser Bäume rund um den Erdball gab.

Wenn der von DARwin geprägte Ausdruck von einem lebenden Fossil eine Berechtigung hat, dann für Wollemia. Andere Koniferen-Gattungen, die ebenfalls als lebende Fossilien bezeichnet werden, sind Araukarien (z. B. Araucaria angustifolia und Araucaria araucana), die nordamerikanischen Mammutbäume (Sequoia sempervirens und Sequoiadendron giganteum) und die in China heimischen Arten Ginkgo biloba und Urweltmammutbaum (Metasequoia glyptostroboides). Es ist schon etwas Besonderes: Wollemia nobilis existiert seit rund 100 Millionen Jahren, blieb seither morphologisch relativ unverändert und hat die Jahrmillionen mit nur wenigen Individuen in einem kleinen Rückzugsareal überdauert. Selbst die Entdeckung Australiens 1605 und nachfolgende Veränderungen von Landschaften, Fauna und Flora überlebte Wollemia unbeschadet. Trotz der Ausweisung von mehreren Nationalparks in Australien und damit verbundene intensive Erforschung dieser Gebiete blieb die Wollemi Pine in verborgenen Canyons so lange unentdeckt.

\section{Verbreitung}

Die Araukariengewächse waren über den früheren Südkontinent (Gondwana) weit verbreitet. Ihre heutigen Vertreter leben im Wesentlichen nur noch auf der Südhalbkugel. Die AraucariaArten haben heute ihre natürliche Verbreitung in Australien, Papua Neuguinea, Neukaledonien, auf den Norfolkinseln und in Südamerika (Nimsch 2011 b). Die Arten der Gattung Agathis sind von Sumatra über die Philippinen bis nach Malesien, Fiji, Queensland (Australien) und Neuseeland verbreitet (ECKENWALDER 2009).

Der Wollemi National Park, in dem die Wollemia wächst, liegt zusammen mit dem Yengo National Park und dem Blue Mountains National Park etwa $200 \mathrm{~km}$ nordwestlich von Sydney und hat eine Größe von rund 500000 ha. Der Nationalpark ist von zahllosen Flüssen durchzogen, die im Laufe der Zeit tiefe Canyons gebildet haben. Es handelt sich um ein schwer zugängliches Gebiet, das auf seinen Hochflächen oft von Buschbränden heimgesucht wird. In einem dieser tiefen Canyons wurde Wollemia entdeckt und bald nach ihrer Entdeckung zum Schutz mit Recht strikt von der Außenwelt abgeriegelt. Die drei kleinen Bestände mit insgesamt nicht mehr als hundert Exemplaren 


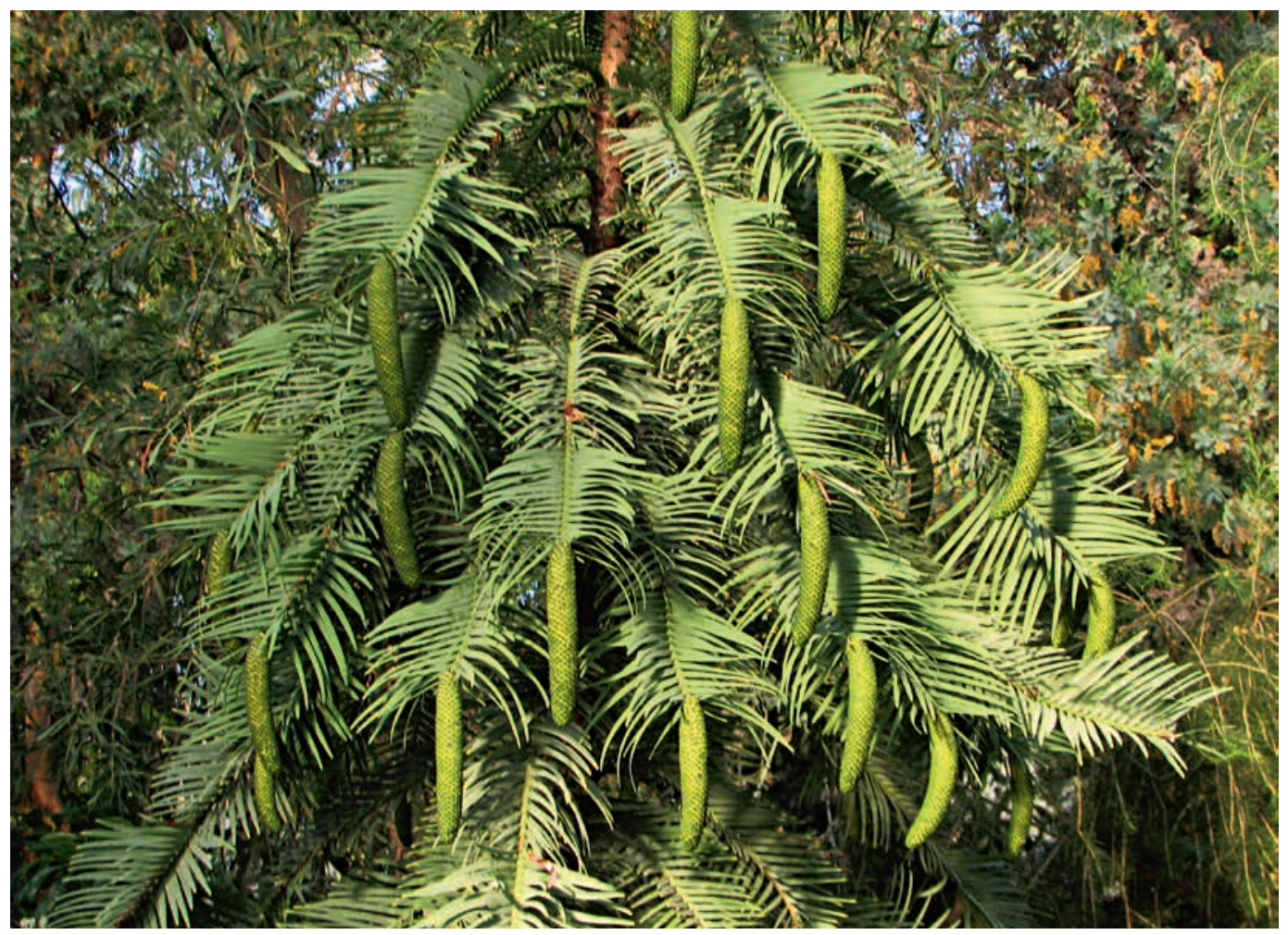

Abb. 4: Wollemia nobilis mit reichlich Pollenzapfen. (Foto: V. M. DöRken)

sollen durch diese Maßnahmen vor schädlichen Einflüssen von außen (z. B. pathogene Pilzsporen) abgeschottet werden. Nur wenige Wissenschaftler dürfen unter Beachtung einiger Schutzvorschriften die Naturstandorte besuchen. Die genauen Standorte bleiben zum Schutz der vom Aussterben bedrohten Art geheim.

\section{Morphologie}

Wollemia nobilis ist ein immergrüner Nadelbaum, der am Naturstandort 40 m Höhe erreicht. In der Regel haben die Kronen einen schlanken, walzenförmigen Habitus. Der Stammdurchmesser erreicht selbst bei einem Alter von ca. 1000 Jahren in Brusthöhe maximal nur 1,2 m. Die Stämme älterer Individuen haben eine auffallende, sehr charakteristische und von vielen kleinen Blasen überzogene Borkenstruktur, die im Alter besonders prominent ausgeprägt ist. Die Verzweigung ist einzigartig. Meist werden nur Seitenzweige der ersten Ordnung ausgebildet. Seitenzweige zweiter Ord-

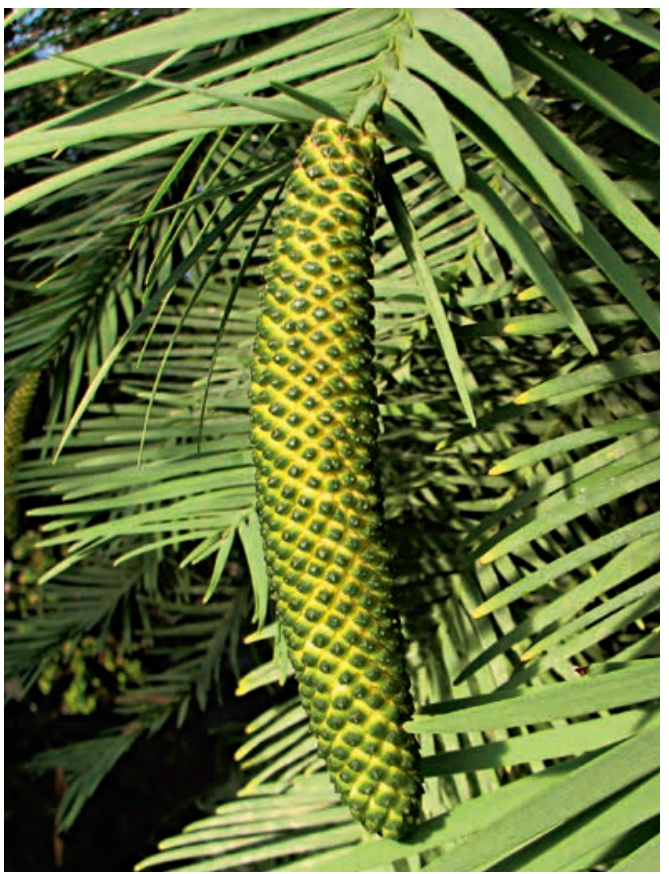

Abb. 5: Pollenzapfen von Wollemia nobilis. (Foto: V. M. DöRKEN) 


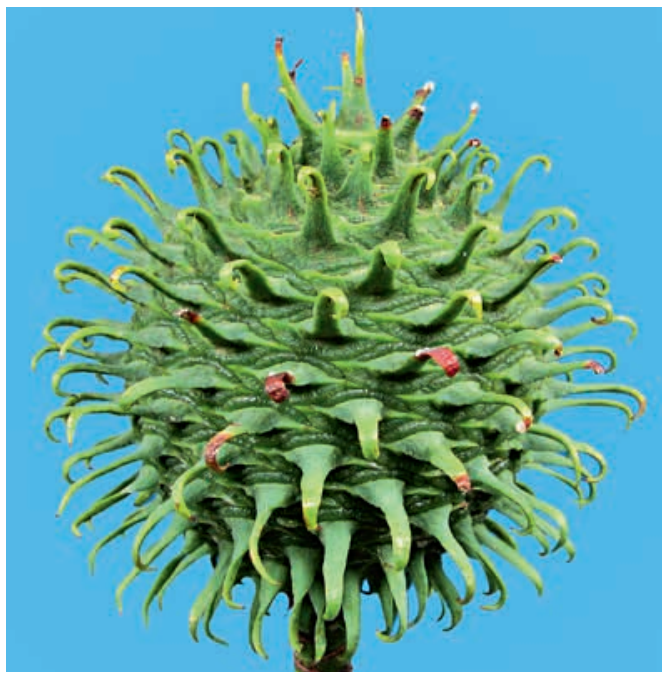

Abb. 6: Weiblicher Zapfen von einem Exemplar im Botanischen Garten Konstanz. (Foto: V. M. DörkeN)

nung findet man in der Regel nur an Trieben, an denen zuvor ein terminaler Zapfen gestanden hat. Nach Abschluss der jährlichen Wachstumsphase werden die großen Knospen (meist die Endknospen) im oberen Kronenbereich mit einem weißen, harzhaltigen Überzug (sogenannte Polarkappen) versehen. Dieser schützt die Knospen bis zum Austrieb vor Frost und anderen Beschädigungen. (Dörken \& Nimsch 2018, 2019).

Die weichen Nadeln inserieren spiralig rings um den Zweig. Sie sind gescheitelt ausgerichtet, wobei auf jeder Seite zwei Blattreihen zu finden sind. Dies vermittelt den Eindruck einer Vierreihigkeit. Die Nadeln haben weder einen deutlichen Blattstiel noch eine verschmälerte Basis. Die Blattbasis läuft die Sprossachse herab, dies erinnert an den Schwanz eines Stegosauriers. Die Nadelblätter werden bis $8 \mathrm{~cm}$ lang und 0,3-1 cm breit. Die Nadelspitze ist abgerundet. Eine deutliche Mittelrippe sowie auffällige Wachszeichnungen fehlen. Die Stomata sind beiderseits in unauffälligen Längsreihen angeordnet, wobei die Dichte der Stomatareihen auf der Nadelunterseite höher als auf der Oberseite ist.

Die reproduktiven Strukturen sind in kompakten, eingeschlechtlichen Zapfen angeordnet. Männliche Pollenzapfen und weiblichen Samen- zapfen werden auf dem gleichen Baum ausgebildet. Sie stehen niemals seitlich, sondern werden immer terminal an der Spitze von Seitentrieben der ersten Ordnung ausgebildet (Dörken \& NimsCH 2018, 2019). Die Samenzapfen sind kugelig und weisen einen Durchmesser von 6-10 cm auf. Sie bauen sich aus zahlreichen Zapfenschuppen auf. Diese sind ein Verwachsungsprodukt aus Deck- und Samenschuppe. Dabei repräsentiert die Deckschuppe ein Tragblatt, in dessen Achsel ein fruchtbarer, jedoch stark kondensierter und reduzierter Kurztrieb (die Samenschuppe) steht. Demzufolge sind die Samenzapfen als verzweigte Blütenstände aufzufassen. Innerhalb eines Zapfenschuppenkomplexes stammen die obersten Gewebeschichten von der Samenschuppe, die untersten von der Deckschuppe (DörkeN \& Rudall 2019). Je Zapfenschuppe wird nur eine Samenanlage gebildet, deren Mikropyle (Bereich, durch den das Pollenkorn ins Innere der Samenanlage gelangt) zur Zapfenspindel zeigt. Die Pollenzapfen sind im Unterschied zu den Samenzapfen unverzweigte Strukturen, die einer Blüte entsprechen. Sie sind bis $15 \mathrm{~cm}$ lang und hängen kätzchenartig. Sie bestehen aus mehreren hundert Pollensäcke tragenden Strukturen (Sporangiophoren). Diese Sporangiophoren sind hyposporangiat: sie bestehen aus einem zentralen Stielchen, einem terminalen Schildchen (Scutellum), das zur Oberseite des Stielchens zeigt sowie zwischen 5 und 10 (seltener 12) Pollensäcken, die nur auf der Unterseite des Stielchens ausgebildet werden (Dörken \& Nimsch 2018, 2019, Dörken \& Rudall 2019).

Wollemia nobilis ist windbestäubt, ein für viele Koniferen typischer Bestäubungstropfen fehlt. Der Pollenfang wird durch eine narbenartig gestaltete Mikropyle übernommen. Nach Freisetzen der Pollenkörner trocknen die Pollenzapfen schnell ein und zerfallen. Die Samenzapfen reifen im zweiten Jahr. Zum Zeitpunkt der Reife zerfallen sie in die einzelnen Schuppen (DöRken \& Nimsch 2018, 2019). Same und Zapfenschuppe sind nicht miteinander verwachsen. Die rhombischen, 1-1,5 $\mathrm{cm}$ langen und 1,5-2 cm breiten braunen Samen sind flach und weisen ringsum einen schmalen Flügelsaum auf. Pro Samenzapfen werden etwa 
300 Samen gebildet. Am Naturstandort keimen diese Samen sehr zahlreich, doch Pilzbefall, Lichtmangel und stark versauerter Boden vernichten die meisten von ihnen wieder. Diese Tatsache und der räumlich begrenzte Standort im Canyon haben eine weitere Ausbreitung des Wollemia-Bestandes verhindert (GRIMSHAW 2009).

\section{Schutz und Vermehrung}

Zur Erhaltung von Wollemia wurden Samen und Stecklinge (auch zur Meristem-Vermehrung) gesammelt, um außerhalb des Naturstandortes weitere Populationen aufzubauen. Auch die Verbreitung der Bäume über den Verkauf von Jungpflanzen wurde durch eine australische Baumschule betrieben und sollte zum künftigen Arterhalt beitragen. Die ersten Jungpflanzen wurden vom Auktionshaus Sotheby's in London versteigert und erbrachten einen Erlös von 1,5 Millionen Dollar. Vertraglich ist geregelt, dass von jedem Verkauf einer Wollemi Pine ein gewisser Betrag der Forschung und der Erhaltung des Naturstandortes zu Gute kommt. Zwischenzeitlich ist die Vermehrung zur einer Routine geworden, denn die Stecklingsvermehrung funktioniert problemlos und die weltweite Vermarktung ebenfalls. Nach den ersten sehr hohen Pflanzenpreisen kann man heutzutage schon für knapp 40 Euro eine Pflanze im Fachhandel erwerben.

Der Wollemia nobilis ist eine ähnliche weltweite Verbreitung zu wünschen, so wie es mit der zunächst als Fossil beschriebenen Metasequoia glyptostroboides geschah, als sie 1944 lebend in Zentral-China wiederentdeckt wurde. Der Naturbestand im Wollemi National Park ist, bedingt durch sein kleines Naturareal, gefährdet und steht unter strengem Schutz. Doch die Zukunft dieses seltenen Baumes scheint durch Schutz und die massenhafte Vermehrung gesichert. Wollemia ist mittlerweile ein fester Bestandteil in sämtlichen botanischen Sammlungen. Es bleibt zu hoffen, dass der Art durch diese Maßnahmen das künftige Überleben gesichert wurde. Erste flächenhafte Anpflanzungen fanden bereits in Australien statt. Auch im Palmengarten wachsen im Freiland zwischen Historischem Eingangsschauhaus und Tropicarium zwei Wollemia-Exemplare. Im Winter

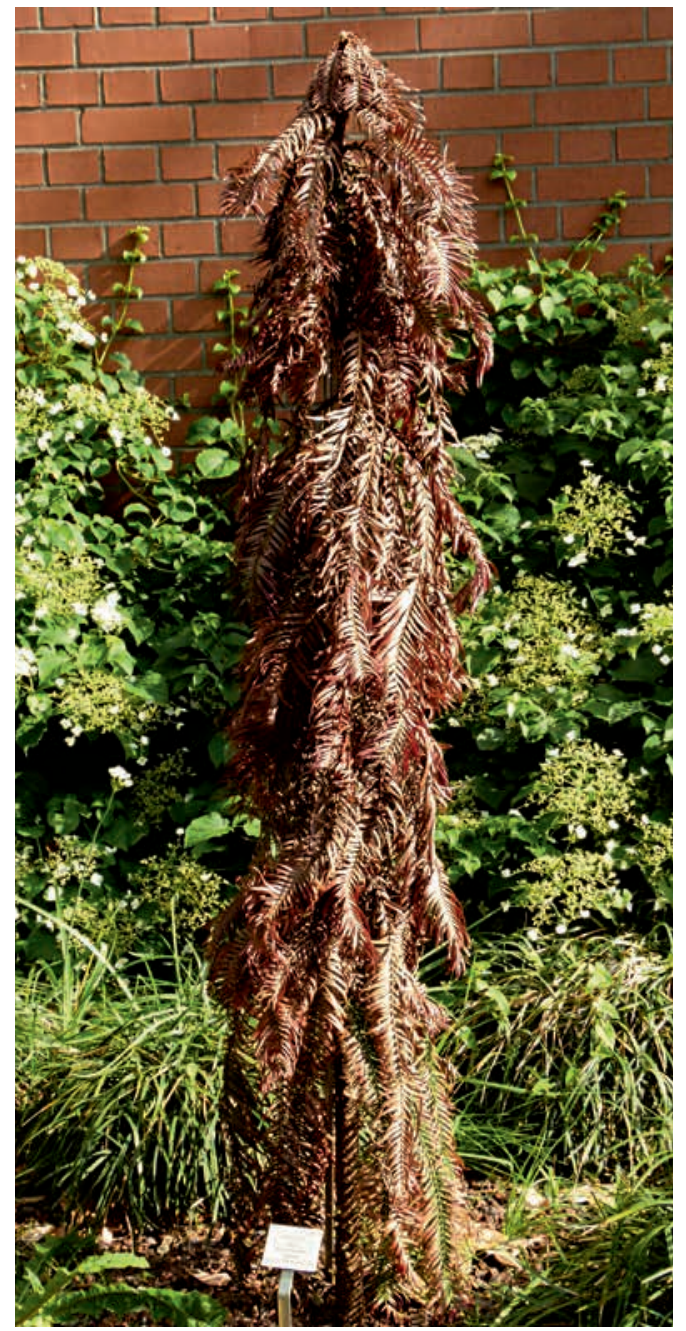

Abb. 7: Scheinbar völlig erfroren sah eine Wollemia nobilis im Palmengarten im Sommer 2012 aus. Das Bäumchen ist aber wieder ausgetrieben und hat sich mittlerweile zu einem prächtigen, rund $3 \mathrm{~m}$ hohen Exemplar entwickelt. (Foto: M. JАСОВI)

werden sie durch Nadelreiser und Vlies geschützt. Mittlerweile werden auch männliche und weibliche Zapfen gebildet.

\section{Standort und Pflege}

Die Temperatur am Naturstandort variiert von $-5^{\circ} \mathrm{C}$ bis $+45^{\circ} \mathrm{C}$. Offensichtlich verträgt Wollemia nach Freiland-Auspflanzungen in Europa, Japan und Amerika sogar doch einige Frostgrade mehr. Wollemia ist in die USDA Hardiness Zone 8-9 


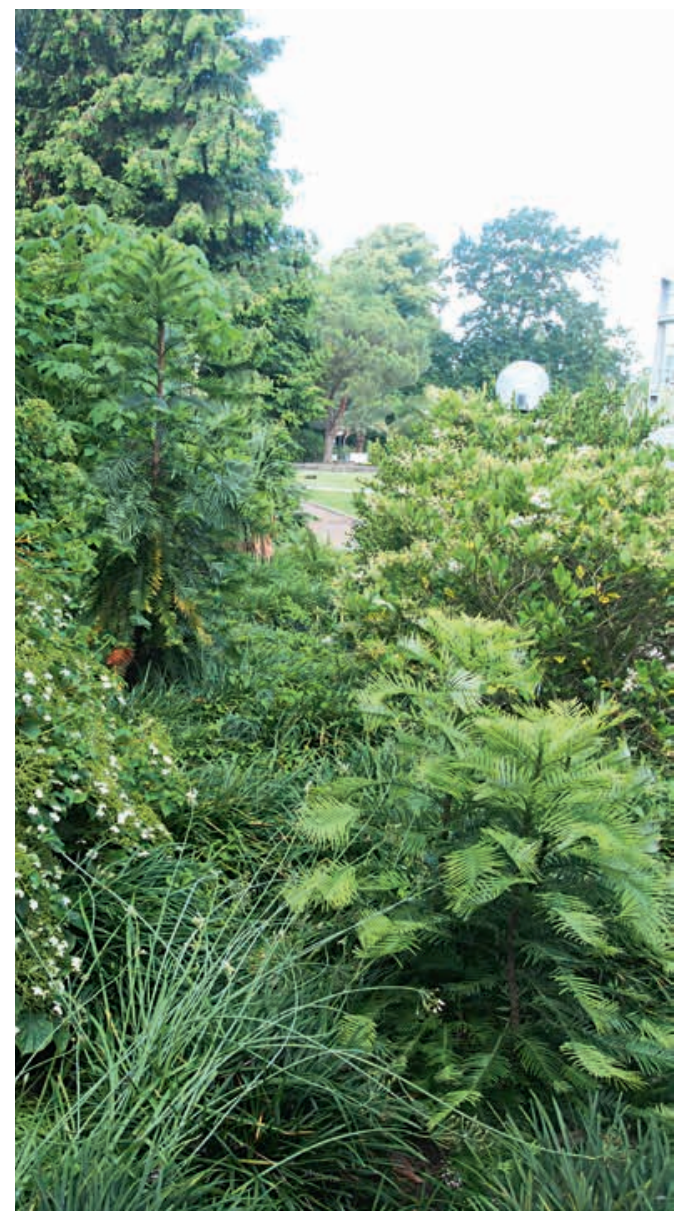

Abb. 8: Bereits zwei Jahre nach dem starken Frostschaden (Juni 2014) hatte die Wollemia im Palmengarten (im Bild das vordere Exemplar) wieder eine schöne Form und zeigte einen üppigen Neuaustrieb. (Foto: H. STEINEcke)

einzustufen (Grimshaw 2009). Im Botanischen Garten Meran (Trauttmansdorff) hat ein etwa $5 \mathrm{~m}$ hohes Exemplar $-12{ }^{\circ} \mathrm{C}$ ohne Schäden überstanden. Daraus darf allerdings nicht der Schluss gezogen werden, dass noch tiefere Minusgrade ohne Schaden möglich sind. Es darf vermutet werden, dass Wollemia nicht zu einem Allerweltsbaum in unseren Parkanlagen und Gärten werden wird, sondern nur an bestimmten, klimatisch besonders bevorzugten Standorten in Deutschland zu einem ansehnlichen kleinen Baum heranwachsen wird (Nimsch 2011). Selbst gut geschützte Exemplare sind im Arboretum Freiburg bei ca. $-10{ }^{\circ} \mathrm{C}$ erfroren. Neue Austriebe aus dem Wurzelstock nach den Frostschädigungen wuchsen auffallend üppig, bedingt durch ein Missverhältnis von Wurzelstock zu (fehlender) Nadelsumme. Im darauffolgenden Winter erfroren die neuen, nicht genügend verholzten Triebe erneut trotz gutem Schutz. Das bedeutet, dass eine größere Winterhärte durch gute Schutzmaßnahmen alleine nicht erreicht werden kann. Möglicherweise können Pflanzen, die mehrere milde Winter überstanden haben, mit zunehmendem Alter etwas stabiler werden. Dagegen sind die Voraussetzungen für eine dauerhafte Etablierung in mediterranen Regionen oder in westeuropäischen Küstengebieten wesentlich günstiger. Nach ersten Auspflanzungen ins Freiland haben botanische Gärten und Privatpersonen in Deutschland keine guten Erfahrungen gemacht. Diese reichen von deutlichen Blattschäden trotz Winterschutz bis hin zu häufigen Totalausfällen infolge Frost. So erfroren selbst am klimatisch ansonsten sehr begünstigten Bodensee in den Extremwintern 2008/2009 sowie 2011/2012 nahezu alle im Freiland wachsenden Wollemia-Bäume bzw. erlitten massivste Frostschäden. Auch das damals einzige ausgepflanzte Exemplar des Palmengartens schien zunächst komplett erfroren zu sein, hat sich danach aber wieder sehr gut erholt und ist jetzt etwa $3 \mathrm{~m}$ hoch. Als Winterschutz wird eine dicke Abdeckung des Wurzelraumes mit Rindenmulch mit einer Reisigpackung oder mit Vergleichbarem empfohlen, damit der Wurzelraum nicht durchfriert und die (eingeschränkte) Assimilation weiter funktionieren kann. Empfohlen wird ein Seitenschutz aus Reisig oder Schilfmatten, der nach oben offen bleibt. Ein völliges Einbinden der immergrünen Pflanze sollte nicht stattfinden. Weitere Erfahrungen mit der Kultur von Wollemia nobilis sind Demes (2019) zu entnehmen.

Bei einer Freilandpflanzung kann bei Beachtung einiger Regeln ein gewisser Erfolg möglich sein. Wie bei chilenischen Araucaria-Pflanzen sollte der Standort sehr gut drainiert sein, wenn möglich an einem Hang liegen, sich von der Wintersonne und austrocknenden Ostwinden abgewandt befinden und einen Seitenschutz aus anderen Bäumen oder einer Mauer haben. Eine Düngung der Freilandpflanzen ab etwa Juli sollte nicht mehr stattfinden, um ein rechtzeitiges Verholzen der Triebe vor dem 


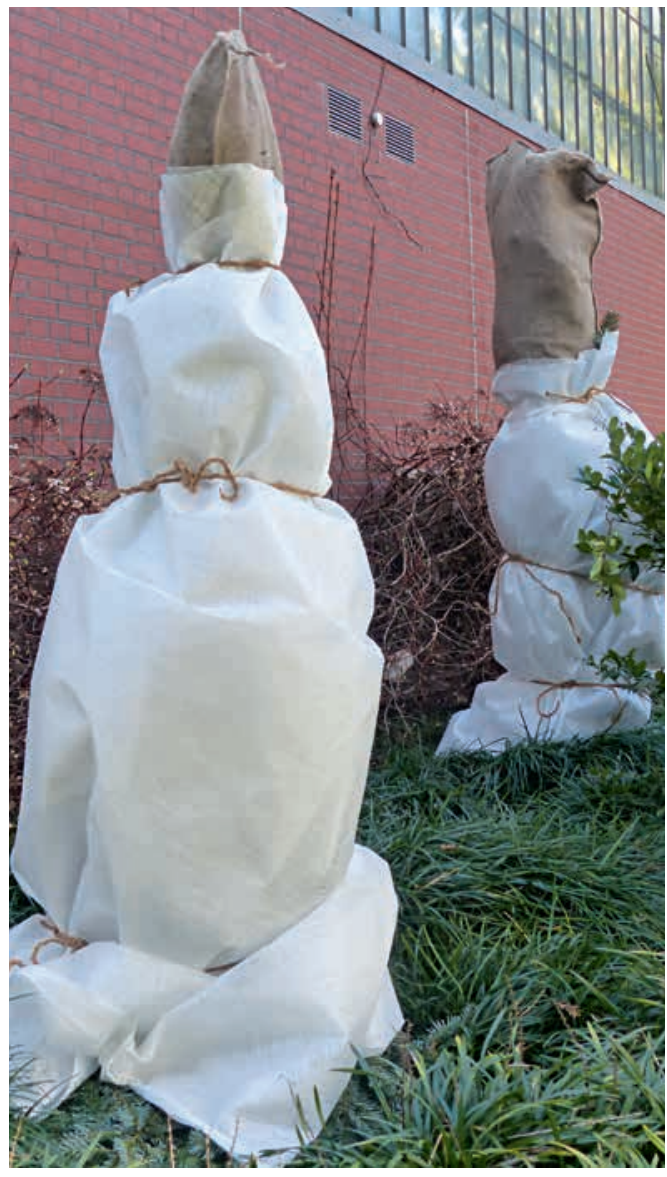

Abb. 9: Winterschutz der beiden Wollemia-Pflanzen im Palmengarten (2018). Aufgrund der milden Witterung im Winter 2019/20 war bis zum Redaktionsschluss keine Verpackung notwendig. (Foto: H. STEINECKE)

Winter zu erreichen. Ungeeignet sind schwere, verdichtete Böden und reine Südlagen wegen der Gefahr der Frosttrocknis. Die Niederschlagsmenge und die Luftfeuchtigkeit sind wesentliche Voraussetzung für die Freilandpflanzung. Bei einer Niederschlagsmenge von unter $1000 \mathrm{~mm}$ fühlen sich Araucaria und Wollemia auf Dauer nicht so wohl (Nimsch 2011). In den letzten beiden trocken-heißen Sommern wurden jedenfalls die Wollemien im Palmengarten reichlich gewässert, was sie mit gutem Wachstum dankten.

Bei der Verwendung als Kübelpflanze sollte ebenfalls eine Drainageschicht (z. B. Blähton oder gekörnte Ziegel) im unteren Topfbereich vorhanden sein, um das Ablaufen überflüssigen Gießwassers zu gewährleisten. Auf keinen Fall darf Staunässe entstehen. Eine Vlieslage zwischen Drainageschicht und Pflanzerde, in die die Wollemie gepflanzt werden soll, verhindert ein Abschwemmen der Erde in die Drainage.

Es bleibt zu hoffen, dass alle Maßnahmen zur Erhaltung dieser seltenen Konifere Erfolg haben und das Überleben dieses australischen Endemits auch künftig gesichert ist.

\section{Literaturverzeichnis}

Demes, B. 2019: Wollemia, wie geht's? - Gp 1/2019: 26-27.

Dörken, V. M. \& Nimsch, H. 2018: Differentialdiagnostik in Koniferen - ein illustrierter Gattungsschlüssel. - RemagenOberwinter.

Dörken, V. M . \& Nimsch, H. 2019: Morphology and identification of coniferous genera. - Remagen-Oberwinter.

Dörken, V. M. \& Rudall, P. 2019: Structure and abnormalities in cones of the Wollemi pine (Wollemia nobilis). - Kew. Bull. 74:3.

FARJon, A. 2008: A natural history of conifers. - Portland.

Grimshaw, J. 2009: New trees. - Richmond.

Nimsch, H. 2011 a: Wollemia nobilis. In: Bouffier, V. A. \& Gandert, K. D. 2011: Beiträge zur Gehölzkunde. - Hemmingen.

Nimsch, H. 2011 b: Araucaria, alle Arten der Gattung in Bild und Text. - Remagen-Oberwinter.

TAylor, T. N.; TAylor, E. L. \& Krings, M. 2009: Paleobotany, the biology and evolution of fossil plants. 2. Aufl. Burlington, London, San Diego, New York.

Woodford, J. 2005: The Wollemi Pine. - Melbourne.

\section{Internetseiten}

https://www.zeit.de/wissen/umwelt/2020-01/australien-feuerkarte-braende-buschfeuer-flaechenbraende-duerre

https://www.spektrum.de/news/buschfeuer-in-australien-sindkatastrophe-fuer-das-oekosystem/1695552

\section{Anschrift der Autoren}

Dr. Veit Martin Dörken, Universität Konstanz, Fachbereich Biologie, Universitätsstraße 10, 78457 Konstanz, E-Mail: veit.doerken@uni-konstanz.de

Hubertus Nimsch, St. Ulrich 31, 79283 Bollschweil, E-Mail: hubertus.nimsch@t-online.de 